\title{
INFLUENCE OF PHYSICAL THERAPY AND CHOLINERGIC MEDICATIONS ON THE LIFE QUALITY PARAMETERS IN POST STROKE PATIENTS WITH THE ARM MOTOR DYSFUNCTION
}

\author{
Iriah Festus Uwa-Agbonikhena
}

Ivano-Frankivsk National Medical University,

Department of Neurology and Neurosurgery, Galytska str., 2, Ivano-Frankivsk, 76018, Ukraine

e-mail: iriahuwagbae@gmail.com

\begin{abstract}
Motor dysfunction makes the greatest contribution to the patient's functional independence and has a significant impact on the quality of life (QOL) of post-stroke patients.

The objective of the research was to evaluate the impact of different physical therapy approaches and choline alphoscerate on the functional independence and quality of life in patients in 1 year after ischemic stroke.

Materials and methods. There were 104 patients examined in 1-year period after first anterior circulation ischemic stroke. Neurological status, functional independence and QOL were assessed according to the National Institute of Health Stroke Scale (NIHSS), modified Rankin Scale (mRS), Functional Independence Measurement (FIM) and Stroke Specific Quality of Life Scale (SS-QOL). Patients in Group 1 received secondary stroke prevention therapy and performed the exercises complex for general muscle function improvement for 2 months; patients in Group 2 also performed the exercise complex for hand motility improvement for 2 months; patients in Group 3 received choline alphoscerate $400 \mathrm{mg}$ twice a day for 2 months in addition to the abovementioned physical therapy.

Results. 2 months of physical therapy in Group 1 resulted in increase of "Transfer" and subtotal motor scores. A significant increase in the "Self-care", "Transfer" and therefore subtotal motor and total scores was observed in the patients in Group 2 and Group 3; the motor subtotal and total scores in Group 3 were $11.4 \%$ and $11.6 \%$ higher than the same indices in Group 1, respectively.

The most significant improvement of QOL parameters was observed in patients in Group 3, whose total score increased by $36.8 \%$ from the baseline $(p<0.05)$, and exceeded the same index of Group 1 by $17.1 \%(p<0.05)$.

Conclusions. Adding of hand exercise into the physical therapy complex for post-stroke patients contributes to better upper extremity motor performance and therefore functional independence. Stimulation of cholinergic neurotransmission with the choline alphoscerate may also increase the efficacy of physical therapy and contribute to patients' general mobility, upper extremity function and QOL.
\end{abstract}

Keywords: stroke, quality of life, physical therapy, functional independence, choline alphoscerate.

Problem statement and analysis of the latest research. Stroke remains one of the leading causes of disability worldwide. A number of disabled post-stroke individuals is constantly rising, mostly due to stroke high incidence and a significant decrease in stroke mortality within the recent decades [13].

Motor dysfunction is the most prevalent and disabling among the stroke consequences. Only $10 \%$ of post-stroke individuals have a full recovery, about $25 \%$ of them recover with minor impairments, and about $40 \%$ of all patients continue to live with moderate disabilities, while $15-30 \%$ live with severe disabilities and are fully dependent [19]. Motor dysfunction makes the greatest contribution to the patient's functional independence, and together with the age, stroke localization and social interactions has a significant impact on the quality of life (QOL) of the patients as well as their caregivers $[1,3,15,21]$

The priority in the treatment of poststroke patients is their rehabilitation and social readaptation. The most effective recovery is possible during the first 6 months after stroke [9]. The leading mechanism of impaired functions recovery is neuroplasticity, which is performed mainly through the mechanisms of synaptic stabilization with changes of the synaptic functional activity, the length and configuration of active zones, new synapses formation, regulation of synaptic transmission efficiency, changes of voltage dependent membrane channels excitability threshold $[9,25]$. Physical activity may stimulate the neuroplasticity $[8,14]$, and benefits of general physical activity for post-stroke patients have been reported $[2,6,22]$, though they have not focused on precise evaluation of its impact on the patients' life quality.

Neuroplasticity and stroke recovery require significant neuronal metabolic reserves, that is why their stimulation could increase the efficacy of the impaired functions recovery. Choline alphoscerate (CA), which decomposes into choline and glycerophosphate within the body, requires attention in the group of neuroprotectors. Free choline is metabolized and then eliminates the acetylcholine insufficiency, causing stimulation of cholinergic synaptic neurotransmission; glycerophosphate is a phosphatidylcholine precursor which promotes stabilization of neuronal and mitochondrial membranes [25]. Studies in the acute and recovery period of stroke [7, 17] demonstrated its favorable influence on the functional outcome; CA has also been reported to be beneficial for cognition in vascular and neurodegenerative diseases due to cholinergic neurotransmition enhancement $[5,23]$.

Although the most active and effective recovery takes place within the early recovery period of stroke, rehabilitation in later periods becomes even more relevant due to delay or inefficiency of the early rehabilitation. That is why research, development and implementation of novel physical therapy tactics becomes a subject of great interest in regard of a prospective of increase of rehabilitation efficacy.

The objective of the research was to evaluate the impact of different physical therapy approaches and CA on 
the functional independence and QOL in patients in 1 year after ischemic stroke.

\section{Materials and methods}

The study was performed at the department of neurology and neurosurgery of Ivano-Frankivsk National Medical University and the vascular neurology department of IvanoFrankivsk Regional Clinical Hospital in 2018-2020. Totally there were 104 patients (62 males, 42 females; mean age constituted $63[53 ; 74]$ years) examined in the 1 -year period after first anterior circulation ischemic stroke. Stroke was localized in the left cerebral hemisphere in $57(54,8 \%)$ patients and in the right hemisphere in 47 (45,2\%) patients.

Exclusion criteria:

1. hemorrhagic stroke;

2. recurrent stroke;

3. posterior circulation stroke;

4. age $<50$ and $>80$;

5. left-handed patients;

6. period of $<12$ month and $>24$ month after stroke;

7. plegia or severe paresis of the upper extremity;

8. modified Rankin Scale (mRS) score $>2$;

9. sensory or severe motor aphasia;

10. malignancy and systemic comorbidities;

11. severe depression (Hospital Anxiety and Depression Scale (HADS) score $>11$ );

12. previous disability (orthopedic, etc).

Neurological status was assessed according to the National Institute of Health Stroke Scale (NIHSS) [4], functional independence was evaluated with the (mRS) [20] and the Functional Independence Measurement (FIM) [16]. QOL was assessed according to the Stroke Specific Quality of Life Scale (SS-QOL) [26]. Screening for depression was performed with the help of HADS scale [27].

Patients were randomized into 3 groups. Patients in Group $1(n=35)$ received secondary stroke prevention therapy and performed the exercise complex for improvement of general muscle function and mobility for 2 months; patients in Group $2(\mathrm{n}=37)$ received secondary stroke prevention therapy, performed the exercise complex for improvement of general muscle function and mobility and also complex of exercises for improvement of the hand motility function for 2 months; patients in Group $3(n=32)$ received secondary stroke prevention therapy, performed the exercise complex for improvement of general muscle function and mobility and also complex of exercises for improvement of the hand motility function and additionally were prescribed CA 400 mg twice a day for 2 months. Patients were examined before and after 2 months of the abovementioned therapy.

Statistical analysis of the results was carried out with the help "MS Excel" and a software environment for statistical calculations "R" (R Core Team RR. Language and environment for statistical computing. R Foundation for statistical computing [Internet]. 2018. Available from: URL: https:// www.r-project.org/) using nonparametric evaluation methods. Data are shown as Median (Me) and interquartile interval [Q25\%; Q75\%]. Statistical significance was defined as p-value $<0.05$.

\section{Results and discussion}

Examination of the patients' baseline neurological status and functional independence (Table 1) showed decreased FIM total and motor subtotal scores due to poor performance in the "Self-care", "Transfer" and "Locomotion" subtests. 2 months of physical therapy in Group 1 resulted into increase of "Transfer" and subtotal motor scores. A significant increase in the "Self-care", "Transfer" and therefore subtotal motor and total scores was observed in patients in Group 2 and Group 3; moreover, the motor subtotal and total scores in Group 3 were $11.4 \%$ and $11.6 \%$ higher than the same indices on Group 1, respectively.

Study of the QOL parameters (Table 2) showed increase in the "Mobility" and total scores in Group 1 by $31.1 \%$ and $14.1 \%$, respectively, in comparison with the baseline. Adding of the hand exercise to the physical therapy complex (Group 2) appeared to be more beneficial for the QOL improvement. After 2 months the patients in Group 2 showed increase in the "Family roles" score by 50.0\% ( $<<0.01)$, "Language" score by $23,1 \%(p<0.05)$, "Mobility" by $46.7 \%(p<0.01)$, "Selfcare" by $54.5 \%(\mathrm{p}<0.01)$, "Social roles" by $25.0 \%(\mathrm{p}<0.05)$, "Upper extremity function" by $45.5 \%(\mathrm{p}<0.01)$ and therefore total score by $27.8 \%(\mathrm{p}<0.05)$ from the baseline level. The "Upper extremity function" score in Group 2 was $23.1 \%$ higher in comparison with the Group $1(\mathrm{p}<0.05)$.

Table 1. Dynamics of the functional independence and neurological status parameters of post-stroke patients, Me [Q25\%; Q75\%]

\begin{tabular}{|c|c|c|c|c|c|c|}
\hline \multirow{3}{*}{ Parameter } & \multicolumn{6}{|c|}{ Score } \\
\hline & \multicolumn{2}{|c|}{ Group 1} & \multicolumn{2}{|c|}{ Group 2} & \multicolumn{2}{|c|}{ Group 3} \\
\hline & Baseline & After 2 months & Baseline & After 2 months & Baseline & After 2 months \\
\hline NIHSS & $8[6 ; 10]$ & $6[4 ; 8]$ & $8[5 ; 10]$ & $6[4 ; 9]$ & $8[5 ; 10]$ & $6[4 ; 8]$ \\
\hline $\mathrm{mRS}$ & $2[1 ; 2]$ & $1[1 ; 2]$ & $2[1 ; 2]$ & $1[1 ; 2]$ & $2[1 ; 2]$ & $1[1 ; 2]$ \\
\hline \multicolumn{7}{|l|}{ FIM parameters (range) } \\
\hline Self-care $(0-42)$ & $27[21 ; 32]$ & $29[24 ; 35]$ & $26[20 ; 33]$ & $33[28 ; 38]^{*}$ & $26[20 ; 32]$ & $36[31 ; 40]^{*}$ \\
\hline Sphincter control (0-14) & $13[11 ; 14]$ & $13[12 ; 14]$ & $13[11 ; 14]$ & $13[12 ; 14]$ & $13[11 ; 14]$ & $13[13 ; 14]$ \\
\hline Transfer $(0-21)$ & $14[12 ; 17]$ & $17[14 ; 19]^{*}$ & $14[12 ; 16]$ & $18[16 ; 19]^{*}$ & $13[11 ; 16]$ & $18[17 ; 19]^{*}$ \\
\hline Locomotion (0-14) & $9[7 ; 12]$ & $11[9 ; 12]$ & $10[7 ; 12]$ & $11[10 ; 12]$ & $10[8 ; 12]$ & $11[10 ; 12]$ \\
\hline Motor subtotal score $(0-91)$ & $63[51 ; 78]$ & $70[59 ; 80]^{*}$ & $63[50 ; 75]$ & $75[66 ; 83]^{*}$ & $62[50 ; 74]$ & $78[66 ; 85]^{* / * *}$ \\
\hline Communication (0-14) & $11[10 ; 13]$ & $11[10 ; 13]$ & $11[10 ; 13]$ & $12[11 ; 13]$ & $11[10 ; 13]$ & $12[11 ; 13]$ \\
\hline Social cognition $(0-21)$ & $14[11 ; 18]$ & $14[11 ; 18]$ & $13[10 ; 17]$ & $15[12 ; 18]$ & $13[10 ; 17]$ & $16[13 ; 19]$ \\
\hline Total (0-126) & $88[72 ; 109]$ & $95[80 ; 111]$ & $87[70 ; 105]$ & $102[89 ; 114]^{*}$ & $86[70 ; 104]$ & $106[90 ; 117]^{* / * *}$ \\
\hline
\end{tabular}

Note: * - significant difference from the baseline level $(\mathrm{p}<0.05)$; ** - significant difference from the Group 1 level $(\mathrm{p}<0.05)$. 
Table 2. Dynamics of the SS-QOL parameters in post-stroke patients, Me [Q25\%; Q75\%]

\begin{tabular}{|c|c|c|c|c|c|c|}
\hline \multirow{3}{*}{ Parameter } & \multicolumn{6}{|c|}{ Score } \\
\hline & \multicolumn{2}{|c|}{ Group 1} & \multicolumn{2}{|c|}{ Group 2} & \multicolumn{2}{|r|}{ Group 3} \\
\hline & Baseline & After 2 months & Baseline & After 2 months & Baseline & After 2 months \\
\hline Energy (3-15) & $8[6 ; 11]$ & $10[8 ; 12]$ & $7[6 ; 11]$ & $10[8 ; 12]$ & $8[6 ; 11]$ & $11[9 ; 13]$ \\
\hline Family roles (3-15) & $7[4 ; 9]$ & $9[6 ; 11]$ & $6[4 ; 9]$ & $9[6 ; 12]^{* *}$ & $7[4 ; 9]$ & $10[7 ; 12]^{* *}$ \\
\hline Language (5-25) & $13[9 ; 18]$ & $14[10 ; 19]$ & $13[9 ; 17]$ & $16[12 ; 21]^{*}$ & $12[9 ; 18]$ & $17[13 ; 22]^{* / * * *}$ \\
\hline Mobility (6-30) & $16[11 ; 22]$ & $21[15 ; 28]^{*}$ & $15[11 ; 23]$ & $22[15 ; 28]^{* *}$ & $15[11 ; 23]$ & $24[18 ; 29]^{* *}$ \\
\hline Mood (5-25) & $14[11 ; 17]$ & $16[12 ; 19]$ & $13[11 ; 17]$ & $16[12 ; 19]$ & $14[11 ; 17]$ & $18[16 ; 21]^{*}$ \\
\hline Personality (3-15) & $9[7 ; 12]$ & $10[8 ; 12]$ & $10[7 ; 12]$ & $11[8 ; 13]$ & $9[7 ; 12]$ & $11[9 ; 12]$ \\
\hline Self-care $(5-25)$ & $12[10 ; 15]$ & $14[11 ; 17]$ & $11[9 ; 14]$ & $17[13 ; 20]^{*}$ & $11[9 ; 14]$ & $17[14 ; 20]^{*}$ \\
\hline Social roles $(5-25)$ & $11[9 ; 13]$ & $12[10 ; 14]$ & $12[9 ; 15]$ & $15[12 ; 17]^{*}$ & $11[9 ; 14]$ & $15[12 ; 18]^{* *}$ \\
\hline Thinking (3-15) & $8[7 ; 10]$ & $8[7 ; 10]$ & $9[7 ; 10]$ & $10[8 ; 12]$ & $8[7 ; 10]$ & $11[8 ; 13]^{*}$ \\
\hline $\begin{array}{l}\text { Upper extremity } \\
\text { function }(5-25)\end{array}$ & $12[9 ; 15]$ & $13[10 ; 17]$ & $11[9 ; 14]$ & $16[12 ; 19] * * / * * *$ & $11[9 ; 15]$ & $18[13 ; 22]^{* * / * * *}$ \\
\hline Vision (3-15) & $10[8 ; 11]$ & $10[8 ; 11]$ & $11[8 ; 11]$ & $10[8 ; 11]$ & $10[8 ; 11]$ & $10[9 ; 11]$ \\
\hline $\begin{array}{l}\text { Work/productivity } \\
(3-15)\end{array}$ & $8[6 ; 10]$ & $9[7 ; 11]$ & $8[6 ; 11]$ & $9[7 ; 11]$ & $9[6 ; 11]$ & $10[8 ; 12]$ \\
\hline Total (49-245) & $128[97 ; 163]$ & $146[112 ; 181]^{*}$ & $126[98 ; 165]$ & $161[121 ; 195]^{* *}$ & $125[96 ; 164]$ & $171[136 ; 205]^{* * / * * *}$ \\
\hline
\end{tabular}

Note: ${ }^{*}$ - significant difference from the baseline level $(\mathrm{p}<0.05) ;{ }^{* *}$ - significant difference from the baseline level $(\mathrm{p}<0.01)$;

$* * *$ - significant difference from the Group 1 level $(\mathrm{p}<0.05)$

The most significant improvement of QOL parameters was observed in patients in Group 3, who received CA in addition to the physical therapy complex. Patients in Group 3 after 2 months showed increase in the "Family roles" score by $42.9 \%(p<0.01)$, "Language" score by $41.7 \%(\mathrm{p}<0.05)$, "Mobility" by 60.0\% ( $<<0.01)$, "Mood" by 28.6\% ( $<<0.05)$, "Self-care" by $54.5 \%(\mathrm{p}<0.01)$, "Social roles" by $34.4 \%$ $(p<0.05)$, "Upper extremity function" by $63.7 \%(p<0.01)$, "Thinking" by $37.5 \%(\mathrm{p}<0.05)$ and therefore total score by $36.8 \%(p<0.05)$ from the baseline level. Moreover, the "Language" and "Upper extremity function" score in Group 3 was $21.4 \%$ and $38.5 \%$ higher, respectively, in comparison with the Group $1(\mathrm{p}<0.05)$; the total score in Group 3 exceeded the same index of Group 1 by $17.1 \%(p<0.05)$.

Patients of all 3 groups demonstrated increase in FIM subtotal motor score in comparison with the baseline level, though this index was significantly higher in Group 2 and 3 because of the upper extremity function parameters. A significant improvement of the FIM total and motor subtotal scores in Group 2 and 3 patients, who performed physical exercise for improvement of both general muscle function and hand function and received CA, was mostly due to increase of the "Self-care" subtest score. This subtest assesses activities of eating, grooming, bathing and toileting, which are provided by the upper extremity. Total FIM score after 2 months significantly increased from the baseline level only in Group 2 and 3, while in Group 1 it did not significantly differ from the baseline level ( $p>0.05$ ). This fact allows us to assume that physical therapy which is targeting upper extremity function is more beneficial for patients' functional independence.

The similar tendency was observed during the evaluation of the QOL dynamics. Patients in Group 2 and 3, who had a significant increase in the FIM score, demonstrated an improvement of the SS-QOL due to increase of the "Family roles", "Language", "Self-care", "Social roles", "Upper extremity function" and "Mobility" scores, while in Group 1 a significant difference was observed only in the "Mobility" subtest. Adding of $\mathrm{CA}$ contributed to an increase of "Thinking" and "Mood" subtests scores in patients of Group 3 , which was not observed in other 2 groups. This fact may be explained by a positive impact of CA on cognition $[5,23]$ and patients' self-perception, which is assessed in these SSQOL subtests.

Impact of general mobility, motor dysfunction and social interactions on the QOL have been reported in recent researches [18, 24], though they have not focused precisely on different parameters of post-stroke motor dysfunction, i.e. upper extremity function. Physical therapy demonstrated its efficacy in the improvement of motor performance and QOL during the first year post-stroke $[6,10,22]$, but its role in later periods has not been established yet. Upper extremity function is also one of the major factors influencing the QOL $[11,12]$ because it plays a leading role in providing the patients' self-care. That is why improvement of its motor performance and therefore the functional independence could also influence the QOL due to decrease in the disability impact on the patients' daily life activities.

Finally, these findings suggest that inclusion of exercise which precisely target hand function and fine motor skills into their physical therapy complex favourably influences their functional independence and therefore the QOL of poststroke patients. Stimulation of cholinergic neurotransmission with the CA may also increase the efficacy of physical therapy and contribute to patients' general mobility, upper extremity function and QOL. 


\section{Prospects of further researches}

Further research with inclusion of larger number of patients, assessment of their functional independence and QOL dynamics after a longer period of time is needed in regard to the prognostic significance of their interactions and impact on the treatment and rehabilitation strategies of poststroke patients.

\section{Conclusions}

1. Adding of exercise for hand function improvement into the physical therapy complex for post-stroke patients contributes to better upper extremity motor performance and therefore is more beneficial for patients' self-care and functional independence.

2. Improvement of the upper extremity motor function favorably influences quality of life mostly due to the SS-QOL "Family roles" $(p<0.01)$, "Language" $(p<0.05)$, "Mobility" $(p<0.01)$, "Self-care" $(p<0.01$, "Social roles" $(p<0.05)$ and "Upper extremity function" ( $\mathrm{p}<0.01)$ scores.

3. Stimulation of cholinergic neurotransmission with the choline alphoscerate may also increase the efficacy of physical therapy and contribute to patients' general mobility, upper extremity function and QOL.

Ethics policy. The study design was assessed and approved by the Ethics Commission of Ivano-Frankivsk National Medical University (Protocol №101/18, 12.04.2018). All experiments involving participants were conducted in accordance with the World Medical Association Declaration of Helsinki. All patients have signed an informed consent before involvement into the study.

Financial disclosure. This research received no specific grant from any funding agency, commercial or not-for-profit sectors.

Conflict of interest: The Authors declare no conflict of interest.

\section{References}

1. Abualait TS, Alzahrani MA, Ibrahim AI et al. Determinants of life satisfaction among stroke survivors 1 year post stroke. Medicine (Baltimore). 2021; 100 (16): e25550. Available from: https://doi. org/10.1097/MD.0000000000025550

2. Blanchet S, Richards CL, Leblond J et al. Cardiorespiratory fitness and cognitive functioning following short-term interventions in chronic stroke survivors with cognitive impairment: a pilot study. International Journal of Rehabilitation Research. 2016; 39 (2): 153-159. Available from: https://doi.org/10.1097/ MRR.0000000000000161

3. Boudokhane S, Migaou H, Kalai A et al. Predictors of Quality of Life in Stroke Survivors: A 1-year Follow-Up Study of a Tunisian Sample. J Stroke Cerebrovasc Dis. 2021; 30 (4): 105600. Available from: https://doi.org/10.1016/j.jstrokecerebrovasdis.2021.105600

4. Brott T, Adams HP, Olinger CPbet al. Measurements of acute cerebral infarction; a clinical examination scale. Stroke. 1989; 20: 864 - 870. Available from: https://doi.org/10.1161/01.str.20.7.864

5. Catanesi M, d'Angelo M, Antonosante A et al. Neuroprotective potential of choline alfoscerate against I-amyloid injury: Involvement of neurotrophic signals. Cell Biol Int. 2020;44 (8): 1734-1744. Available from: https://doi.org/10.1002/cbin.11369

6. Debreceni-Nagy A, Horvбth J, Bajusznй Kovбcs N. et al.
The effect of low-intensity aerobic training on cognitive functions of severely deconditioned subacute and chronic stroke patients: a randomized, controlled pilot study. International Journal of Rehabilitation Research. 2019; 42 (3); 275-279. Available from: https://doi.org/10.1097/MRR.0000000000000346

7. Gerasymchuk VR. Indicators of energy metabolism in patients during the early recovery period of ischemic stroke under the influence of complex therapy using neuroprotectors and vasoactive drugs. Archive of Clinical Medicine. 2016; Vol. 22, №1: 61 - 65 .

8. Han P, Zhang W, Kang L et al. Clinical Evidence of Exercise Benefits for Stroke. Advances in Experimental Medicine and Biology. 2017; 1000: 131-151. https://doi.org/10.1007/978-981-10-4304-8_9

9. Hara Y. Brain plasticity and rehabilitation in stroke patients. Journal of Nippon Medical School. 2015; 82 (1): 4 - 12. Available from: https://doi.org/10.1272/jnms.82

10. Hsieh HC, Liao RD, Yang TH et al. The clinical effect of Kinesio taping and modified constraint-induced movement therapy on upper extremity function and spasticity in patients with stroke: a randomized controlled pilot study [published online ahead of print, 2021 Jan 15]. Eur J Phys Rehabil Med. 2021;10.23736/S19739087.21.06542-4. Available from: https://doi.org/10.23736/S19739087.21.06542-4

11. Johnston TE, Keller S, Denzer-Weiler C, Brown L. A Clinical Practice Guideline for the Use of Ankle-Foot Orthoses and Functional Electrical Stimulation Post-Stroke. J Neurol Phys Ther. 2021; 45(2): 112-196. Available from: https://doi.org/10.1097/ NPT.0000000000000347

12. Kanai M, Izawa KP, Kubo H, et al. Association of Health Utility Score with Physical Activity Outcomes in Stroke Survivors. Int J Environ Res Public Health. 2020; 18 (1): 251. Available from: https://doi.org/10.3390/ijerph18010251

13. Krishnamurthi RV, Moran AE, Feigin VL et al. Stroke prevalence, mortality and disability-adjusted life years in adults aged 20-64 years in 1990-2013: data from the global burden of disease 2013 study. Neuroepidemiology. 2015; 45: 190-202. Available from: https://doi.org/10.1159/000441098

14. Leisman G, Moustafa AA, Shafir T. Thinking, Walking, Talking: Integratory Motor and Cognitive Brain Function. Frontiers in Public Health. 2016; 4: 94. Available from: https://doi.org/10.3389/ fpubh.2016.00094

15. Lin C, Lee J, Hurt CP et al. Gait Measures at Admission to Inpatient Rehabilitation after Ischemic Stroke Predict 3-Month Quality of Life and Function. PM R. 2021; 13 (3): 258-264. Available from: https://doi.org/10.1002/pmrj.12402

16. McDowell I, Newell,C. Measuring health: a guide to rating scales and questionnaires (2nd Ed.). New York, Oxford University Press. 1996: 63-67.

17. PayenokAV, ZadorozhnaBV, KukhlenkoOYaetal. Influence of Gliatilin on the Dynamics of Neuropsychological Functions and Systemic Inflammatory Response Parameters in Patients in the Recovery Period of Ischemic Stroke. International Neurological Journal. 2013; 8 (62): 51-57 [In Ukrainian].

18. Pedersen SG, Friborg O, Heiberg GA et al. Stroke-Specific Quality of Life one-year post-stroke in two Scandinavian countryregions with different organisation of rehabilitation services: a prospective study. Disabil Rehabil. 2020; 1-11. Available from: https://doi.org/10.1080/09638288.2020.1753830

19. Rancic NK, Mandic MN, et al. Health-Related Quality of Life in Stroke Survivors in Relation to the Type of Inpatient Rehabilitation in Serbia: A Prospective Cohort Study. Medicina (Kaunas). 2020; 56 (12): 666. Available from: https://doi.org/10.3390/medicina56120666

20. Rankin J. Cerebral vascular accidents in patients over the age of 60. Scottish Medical Journal. 1957; 2: 200-215.

21. Sadlonova M, Wasser K, Nagel J et al. Health-related quality of life, anxiety and depression up to 12 months post-stroke: Influence of sex, age, stroke severity and atrial fibrillation - A longitudinal 
subanalysis of the Find-AFRANDOMISED trial. J Psychosom Res. 2021; 142: 110353. Available from: https://doi.org/10.1016/j. jpsychores.2020.110353

22. Tollor J, Nagy F, Csutorøs B et al. High Frequency and Intensity Rehabilitation in 641 Subacute Ischemic Stroke Patients. Arch Phys Med Rehabil. 2021; 102 (1): 9-18. Available from: https:// doi.org/10.1016/j.apmr.2020.07.012

23. Traini E, Carotenuto A, Fasanaro AM et al. Volume Analysis of Brain Cognitive Areas in Alzheimer's Disease: Interim 3-Year Results from the ASCOMALVA Trial. J Alzheimers Dis. 2020; 76(1): 317-329. Available from: https://doi.org/10.3233/JAD-190623

24. van Mierlo ML, van Heugten CM, Post MW et al. Quality of Life during the First Two Years Post Stroke: The Restore4Stroke Cohort Study. Cerebrovasc Dis. 2016; 41(1-2): 19-26. Available from: https://doi.org/10.1159/000441197
25. Vinogradov OI, Daminov VD, Rybalko NV. Use of choline alphoscerate (Gliatiline) in patients with ischemic stroke. Journal of Neurology and Psychiatry n. a. S. S. Korsakov. 2013; №1: 43 - 45 [in Russian].

26. Williams LS, Weinberger M, Harris LE et al. Development of a stroke-specific quality of life scale. Stroke. 1999; 30 (7): 13621369. Available from: https://doi.org/10.1161/01.str.30.7.1362

27. Zigmond AS, Snaith RP. The Hospital Anxiety and Depression Scale. Acta Psychiatrica Scandinavica. 1983; 67 (6): 61-370. Available from: https://doi.org/10.1111/j.1600-0447.1983. tb09716.x

Received: 01.04.2021

Revised: 24.05.2021

Accepted: 25.05.2021 